Pacific Journal of Mathematics

ANALYTIC CONTINUATION OF LOCAL REPRESENTATIONS
OF LIE GROUPS 


\title{
ANALYTIC CONTINUATION OF LOCAL REPRESENTATIONS OF LIE GROUPS
}

\author{
Palle E. T. Jorgensen
}

\begin{abstract}
We consider a symmetric space $(G, K, \sigma)$ where $G$ is a Lie group, $K$ a closed subgroup, and $\sigma$ the involutive automorphism defining the space. A local representation $\pi$ is defined for $g$ in a neighborhood of $e$ in $G$, and the operator $\pi(g)$ is unbounded and defined on a dense subspace in a Hilbert space where the identity

$$
\pi\left(g^{-1}\right)=\pi(\sigma(g))^{*}
$$

holds. We study analytic continuations of $\pi$ to unitary representations of a group $G^{*}$ which is dual to $G$.
\end{abstract}

1. Introduction. Recent papers by Klein and Landau [12], and Fröhlich-Osterwalder-Seiler [4] have studied local representations of Lie groups in connection with Euclidean quantum field theory. See also [16, 19, 21]. Important physical ideas seem to have originated in part with the earlier Lüscher-Mack paper [13]. Earlier mathematical papers where local representations and analytic continuation played an important role, are [6] and [18]. The representations in question have become known as "virtual representations" of symmetric spaces [4]. In these earlier papers, only particular symmetric spaces were considered, and a technical spectral condition was imposed. In a fourth paper, by the author [8], the case of general symmetric spaces was solved, and, moreover, the spectral condition was removed completely. Integrability of Lie algebras of unbounded operators [9] was used systematically, for the first time, in solving the analytic continuation problem. As a consequence of this, the existence theorems were established in a more general setting dictated by particular models.

Let $G$ be a Lie group, $K$ a closed subgroup, and $\sigma$ an involutive automorphism with $K$ as fixed point subgroup. If $\mathfrak{g}=\mathfrak{f}+\mathfrak{m}$ is the corresponding real symmetric Lie algebras we form $\mathrm{g}^{*}=\mathfrak{f}+i \mathrm{~m}$ which is also a real Lie algebra, and let $G^{*}$ denote the simply connected real Lie group with $\mathrm{g}^{*}$ as Lie algebra. R. Schrader suggested to the author [19] that the suitable setting for local representations $\pi$ of the symmetric space $(G, K, \sigma)$ is as follows: A complex Hilbert space $\mathscr{H}$, with dense linear subspace $\mathscr{D}$, is fixed at the outset. A local representation $\pi$ of a neighborhood $U$ of $e$ in $G$ is given, and it is assumed that $\pi(g)$ is defined (possibly 
unbounded) on $\mathscr{D}$ for all $g \in U \subset G$, and

$$
\pi\left(g^{-1}\right) \subset \pi(\sigma(g))^{*}, \quad g \in U .
$$

In earlier work $[4,8,12]$ it was assumed further that $U$ is invariant under translation to the right by elements $k$ in the subgroup $K$, i.e., $U \cdot K=U$, and $\pi(k) \mathscr{D}=\mathscr{D}, k \in K$. In this paper, we show that this global assumption may be replaced by a purely local one which was dictated by particular models and suggested by Schrader, and the integrability conclusion for the analytically continued representation $\pi^{*}$ of $G^{*}$ may still be preserved.

The construction of $\pi^{*}$ is carried out in two steps. First an infinitesimal representation, $d \pi$ of $\mathrm{g}$, is constructed from $\pi$ by differentiation, generalizing classical ideas going back to Segal and Gårding [9]. Starting with $d \pi$, we define a second infinitesimal representation, $d \pi^{*}$ of $\mathrm{g}^{*}=\mathfrak{f}$ $+i \mathrm{~m}$, by

$$
d \pi^{*}(x+i y)=d \pi(x)+i d \pi(y), \quad \text { on } \mathscr{D}, \text { for } x \in \mathfrak{H}, y \in \mathfrak{m} .
$$

In the second step we prove, using a main result from [5], that the representation $d \pi^{*}$ of $\mathrm{g}^{*}$ exponentiates to a strongly continuous unitary representation $\pi^{*}$ of $G^{*}$ on $\mathscr{H}$. We recall that $\pi^{*}$ may be viewed as an analytic continuation because there are self-adjoint operators $H$ and $M$, defined on $\mathscr{D}$ such that $i H$ extends the skew-hermitian operator $d \pi(x)$, and similarly $M$ extends the hermitian operator $d \pi(y)$. If exp: $\mathrm{g}^{*} \rightarrow G^{*}$ denotes the exponential mapping of Lie theory [7], then for $t \in \mathbf{R}$, we have

$$
\pi^{*}(\exp (t x) \exp (i t y))=e^{i t H} e^{i t M},
$$

while $e^{i t H}$ extends $\pi(\exp t x)$, and $e^{t M}$ (which is generally unbounded) extends $\pi(\exp t y)$.

This paper originated with suggestions by R. Schrader [19], which he kindly communicated to us after receiving [8] in preprint form. Applications will appear in [20].

We are extremely grateful to Professor Schrader for asking us to write up the result, and giving permission to quote him. We also wish to thank Professor W. H. Klink for useful suggestions during the author's talk in the math.-physics seminar at the University of Iowa.

2. Local representations. Let $G$ be a Lie group with Lie algebra $\mathfrak{g}, K$ a closed subgroup, and $\sigma$ an involutive automorphism of $G$ such that

$$
\left(K_{\sigma}\right)_{0} \subset K \subset K_{\sigma}
$$


where $K_{\sigma}=\{g \in G: \sigma(g)=g\}$, and $\left(K_{\sigma}\right)_{0}$ is the connected component of the identity $e$ in $K_{\sigma}$. We say that $(G, K, \sigma)$ is a symmetric space [7]. Let $d \sigma: \mathfrak{g} \rightarrow \mathfrak{g}$, be the corresponding infinitesimal Lie automorphism. Then

$$
\mathfrak{f}=\{x \in \mathfrak{g}: d \sigma(x)=x\}
$$

is a sub-Lie algebra, and it is the Lie algebra of $K$. If

$$
\mathfrak{m}=\{y \in \mathfrak{g}: d \sigma(y)=-y\},
$$

then

$$
\mathfrak{g}=\mathfrak{f}+\mathfrak{m} \quad \text { (direct sum of real vector spaces), }
$$

and

$$
\begin{aligned}
& {[\mathfrak{f}, \mathfrak{l}] \subset \mathfrak{H},} \\
& {[\mathfrak{H}, \mathfrak{m}] \subset \mathfrak{m},} \\
& {[\mathfrak{m}, \mathfrak{m}] \subset \mathfrak{H} .}
\end{aligned}
$$

Then form $\mathrm{g}^{*}=\mathfrak{i}+i \mathrm{~m}$, again defined as a direct sum of real vector spaces. It is easy to check that $\mathrm{g}^{*}$ is again a real Lie algebra. By Ado's theorem [7], there is a simply connected Lie group $G^{*}$ which has $\mathrm{g}^{*}$ as its Lie algebra. Moreover, $G^{*}$ is unique up to Lie isomorphism.

Following [12] and [4], we shall consider a local homomorphism $\pi$ of a neighborhood $U$ of $e$ in $G$ such that $\pi(g)$ is a densely defined operator on a complex Hilbert space $\mathscr{H}$ for all $g \in U$. The restriction $\left.\pi\right|_{U \cap K}$ is, of course, a local representation of the subgroup $K$, and we shall assume that $\left.\pi\right|_{U \cap K}$ extends to a strongly continuous unitary representation, also denoted by $\pi$, of $K$ on $\mathscr{H}$. The following technical assumptions [LR1-3] will be part of our defining properties for a local representation $\pi$ of the symmetric space $(G, K, \sigma)$ :

(LR1) For all $g \in U$, the space $\mathscr{D}$ is contained in the domain $\mathscr{D}(\pi(g))$ of the operator $\pi(g)$.

(LR2) If $g_{1}, g_{2}$, and $g_{1} \cdot g_{2}$ are in $U$, and $\psi$ in $\mathscr{D}$, then $\pi\left(g_{2}\right) \psi \in$ $\mathscr{D}\left(\pi\left(g_{1}\right)\right)$, and

$$
\pi\left(g_{1}\right) \pi\left(g_{2}\right) \psi=\pi\left(g_{1} \cdot g_{2}\right) \psi .
$$

(LR3) If $y \in \mathfrak{m}$, and $\exp y \in U$, then $\exp t y \in U$ for all $t, 0 \leq t \leq 1$, and

$$
\lim _{t \rightarrow 0} \pi(\exp t y) \psi=\psi
$$

for all $\psi \in \mathscr{D}$.

While it was assumed in [4] that $U$ is globally invariant under right-translations by all elements $k$ in $K$, and $\mathscr{D}$ is $\pi(k)$ invariant, we drop this assumption on $U$, and replace it by two purely local conditions. 
By virtue of $[3,11]$, and (2.4) and (2.5) above, we have a local hermitian semigroup $\{\pi(\exp t y)\}$ of unbounded operators in $\mathscr{H}$ for each $y \in \mathfrak{m}$. It follows that, for some self-adjoint operator $M$, with $\mathscr{D}(M)$ containing $\mathscr{D}$, we have the operator inclusion

$$
\pi(\exp t y) \subset e^{t M},
$$

for $t$ sufficiently small, where the right hand side $e^{t M}$ is self-adjoint and defined by the spectral theorem applied to $M$. The restriction of $M$ to $\mathscr{D}$ will be denoted by $d \pi(y)$. It follows that every local representation $\pi$ satisfying (LR1-3) has an infinitesimal representation $d \pi$ of the Lie algebra $\mathrm{g}$, and, moreover, the construction of $d \pi$ in this context generalizes the Garding construction from [2]. Our construction of $d \pi$ uses the nontrivial part of the Extension Theorem for local semigroups of hermitian unbounded operators in Hilbert space [11], see also [3, 13].

In addition to (LR1-3), we shall assume that

(LR4) $\pi(y) \mathscr{D} \subset \mathscr{D}, y \in \mathfrak{m}$. Moreover,

(LR5) For each $\psi \in \mathscr{D}$, there is a neighborhood $V_{\psi}$ of $e$ in $K$ such that $U \cdot V_{\psi} \subset U^{2}$, and

$$
\pi(k) \psi \in \mathscr{D}, \quad k \in V_{\psi} .
$$

Finally, it is assumed that

(LR6) For $y \in \mathfrak{m}$, and $\psi \in \mathscr{D}$, the function

$$
k \rightarrow \pi\left(\exp \left(A d_{k} y\right)\right) \psi
$$

is locally integrable on the subset

$$
\left\{k \in K: \exp \left(\operatorname{Ad}_{k} y\right) \in U\right\} .
$$

Recall that Ad denotes the usual adjoint representation [7] of $G$ or $g$.

We shall say that $\pi$ is a local representation of $(G, K, \sigma)$ if (LR1-6) are satisfied. If the local representation $\pi^{*}$ constructed in $\S 1$ from $\pi$ extends to a strongly continuous unitary representation of $G^{*}$ or $\mathscr{H}$, then we shall say that $\pi$ can be analytically continued.

From [9] we have the notion of integrability for representations of Lie algebras by unbounded operators. See also $[14,22]$. A representation $d \pi^{*}$ of the Lie algebra $g^{*}$ was recalled in $\S 1$. It is easy to show that a given local representation $\pi$ of $(G, K, \sigma)$ can be analytically continued to a strongly continuous unitary representation $\pi^{*}$ of $G^{*}$ if and only if $d \pi^{*}$ is integrable, and in this case, $\pi^{*}$ may be constructed as an exponential of $d \pi^{*}$. We refer to [9] and [8] for details regarding integrability and local representations. 
Our main result below generalizes earlier work [12] and [4] in three different directions, as described in the Introduction.

THEOREM. Let $\pi$ be a local representation of a symmetric space $(G, K, \sigma)$. Then $\pi$ can be analytically continued to a strongly continuous unitary representation $\pi^{*}$ of $G^{*}$.

3. A subgroup of $G^{*}$. We start with a symmetric space $(G, K, \sigma)$ and consider the corresponding real symmetric Lie algebra $\mathfrak{g}=\mathfrak{f}+\mathfrak{m}$ as in (2.2)-(2.3). Let $\mathfrak{f}_{1}$ denote the smallest Lie subalgebra of $\mathfrak{f}$ which contains $[\mathfrak{m}, \mathfrak{m}]=\operatorname{span}_{\mathbf{R}}\left\{\left[y_{1}, y_{2}\right]: y_{1}, y_{2} \in \mathfrak{m}\right\}$. Then it follows that $\mathfrak{g}_{1}=\mathfrak{f}_{1}+\mathfrak{m}$ is again a symmetric Lie algebra, and moreover that $\mathfrak{f}_{1}$ is an ideal in $\mathfrak{f}$, and $\mathfrak{g}_{1}$ is an ideal in $g$.

We now let $K_{1}$ be the smallest closed subgroup of $K$ which contains the set $\left\{\exp x: x \in \mathbb{F}_{1}\right\}$; and similarly we denote by $G_{1}^{*}$ the closed subgroup of $G^{*}$ which is generated by $\mathrm{g}_{1}^{*}=\mathfrak{f}_{1}+i \mathrm{~m}$. Recall that $G_{1}^{*}$ is the smallest closed subgroup of $G^{*}$ which contains the $\operatorname{set}\{\exp z$ : $\left.z \in \mathfrak{g}_{1}^{*}\right\}$. Since $\mathfrak{f}_{1}$ is an ideal in $\mathfrak{f}$, and $\mathfrak{g}_{1}^{*}$ an ideal in $\mathfrak{g}^{*}$, it follows that the subgroup $K_{1}$ is normal in $K$, while $G_{1}^{*}$ is normal in $G^{*}$. Moreover, $\left(G_{1}^{*}, K_{1}, \sigma^{*}\right)$ is a symmetric space.

We shall make use of the following,

Observation 1. $\mathrm{g}_{1}^{*}$ is the smallest real Lie subalgebra of $\mathrm{g}^{*}$ which contains the subset, $i \mathfrak{m}=\{i y: y \in \mathfrak{m}\}$.

Proof. Let $\mathfrak{h}$ be the smallest real Lie subalgebra of $\mathfrak{g}^{*}$ which contains $i \mathrm{~m}$. Since $\mathfrak{h}$ is closed under the Lie bracket of $\mathfrak{g}^{*}$, it follows that

$$
\left[i y_{1}, i y_{2}\right]=-\left[y_{1}, y_{2}\right] \in \mathfrak{h} \text {, }
$$

for all $y_{1}, y_{2} \in \mathfrak{m}$. Using again that $\mathfrak{h}$ is a Lie algebra, we conclude from this that $\mathfrak{f}_{1} \subset \mathfrak{h}$. Since $i \mathfrak{m} \subset \mathfrak{h}$, it follows that $\mathfrak{g}_{1}^{*}=\mathfrak{f}_{1}+i \mathfrak{m} \subset \mathfrak{h}$.

On the other hand, $g_{1}^{*}$ is a real Lie subalgebra of $g^{*}$ and it contains $i \mathrm{~m}$. Hence $\mathfrak{h} \subset \mathfrak{g}_{1}^{*}$ since $\mathfrak{h}$ is the smallest such real Lie subalgebra.

Let $\pi$ be a local representation of $(G, K, \sigma)$, and let $d \pi$ be the corresponding infinitesimal representation of the Lie algebra $g$ of $G$.

Obersvation 2. For $z=x+i y \in \mathfrak{f}_{1}+i \mathfrak{m}=\mathfrak{g}_{1}^{*}, x \in \mathfrak{f}_{1}, y \in \mathfrak{m}$, we define

$$
d \pi^{*}(z)=d \pi(x)+i d \pi(y)
$$


and it follows that $d \pi^{*}$ is a representation of the real Lie algebra $\mathfrak{g}_{1}^{*}$ such that

$$
\mathscr{D} \subset \mathscr{D}\left(d \pi^{*}(z)\right) \text { for all } z \in \mathfrak{g}_{1}^{*}
$$

and

$$
d \pi^{*}(z) \mathscr{D} \subset \mathscr{D}
$$

Moreover,

$$
\begin{array}{ll}
\left\langle d \pi^{*}(z) \psi_{1}, \psi_{2}\right\rangle=-\left\langle\psi_{1}, d \pi^{*}(z) \psi_{2}\right\rangle & \text { for } z \in \mathrm{g}_{1}^{*} \\
& \text { and } \psi_{1}, \psi_{2} \in \mathscr{D}
\end{array}
$$

where $\langle\cdot, \cdot\rangle$ denotes the inner product of $\mathscr{H}$.

Proof. Recall that, for every $y \in \mathfrak{m}$, we have a local semigroup, $S_{t}=\pi(\exp t y)$ of hermitian operators defined on $\mathscr{D}$ for $t$ sufficiently small. Properties (LR1-3) are used for this. The reader is referred to [11] and [3] for the theory of local semigroups. The Extension Theorem for such semigroups provides us with a self-adjoint operator $M$ such that $S_{t} \subset e^{t M}$ where $\subset$ refers to the usual inclusion for unbounded operators. By virtue of properties (LR3 and 4), it follows that $\mathscr{D}$ is contained in the domain of $M$, and by [8, Lemma A], $\mathscr{D}$ is a core for $M$, and for $e^{t M}$ when $|t|$ is sufficiently small. It follows that the limit

$$
\lim _{t \rightarrow 0} t^{-1}\left(S_{t} \psi-\psi\right)
$$

exists for all $\psi \in \mathscr{D}$. We shall use the terminology

$$
d \pi(y) \psi=\left.\frac{d}{d t} S_{t} \psi\right|_{t=0} .
$$

We have seen that $d \pi(y) \subset M$, and $d \pi(y)=\left.M\right|_{\mathscr{D}}$. Since $M$ is self-adjoint with $\mathscr{D}$ as a core, it follows that $d \pi(y)$ is essentially self-adjoint as an operator with domain $\mathscr{D}$.

We now turn to the infinitesimal operators $d \pi(x)$ for $x \in \mathfrak{f}_{1}$. Recall that $\left.\pi\right|_{K}$, and therefore also $\left.\pi\right|_{K_{1}}$, is a unitary representation, so the operator $d \pi(x)$ is skew-adjoint and defined for vectors $\psi \in \mathscr{H}$ such that the limit

$$
\lim _{t \rightarrow 0} t^{-1}(\pi(\exp t x) \psi-\psi)
$$

exists, and

$$
d \pi(x) \psi=\left.\frac{d}{d t} \pi(\exp t x) \psi\right|_{t=0}
$$


We claim that $\mathscr{D} \subset \mathscr{D}(d \pi(x))$ and $d \pi(x) \mathscr{D} \subset \mathscr{D}$. It is enough to consider elements $x$ in $\mathfrak{f}_{1}$ of the form $x=\left[y_{1}, y_{2}\right]$, for $y_{1}, y_{2} \in m$. We then have

$$
\left[\exp \left(-\frac{t}{n} y_{1}\right) \exp \left(-\frac{t}{n} y_{2}\right) \exp \left(\frac{t}{n} y_{1}\right) \exp \left(\frac{t}{n} y_{2}\right)\right]^{n^{2}}=\exp \left(t^{2} x+\mathcal{O}\left(\frac{1}{n}\right)\right)
$$

as an identity in the group $G$, cf. [7, Lemma 2.4]. For $n$ sufficiently large and $|t|$ small, both sides of the formula are in the neighborhood $U$. Using (LR2), and self-adjoint operators $M_{i}$ associated to $d \pi\left(y_{i}\right), i=1,2$, we then get

$$
\begin{aligned}
& {\left[e^{-i t M_{1} / n} e^{-i t M_{2} / n} e^{i t M_{1} / n} e^{i t M_{2} / n}\right]^{n^{2}} \psi} \\
& =\pi\left(\exp \left(-t^{2} x+\mathcal{O}\left(\frac{1}{n}\right)\right)\right) \psi, \quad \text { for } \psi \in \mathscr{D} .
\end{aligned}
$$

By virtue of [8, Lemma A], and the Trotter-Chernoff-Nelson theorem [15, Theorem 7], we conclude that the limit exists for $n \rightarrow \infty$, and equals $e^{-i t^{2}\left[\overline{M_{1}, M_{2}} \psi\right.} \psi$. It follows that $\psi \in \mathscr{D}(d \pi(x))$, and

$$
\begin{aligned}
d \pi(x) \psi & =-\overline{\left[M_{1}, M_{2}\right]} \psi=-\left[M_{1}, M_{2}\right] \psi \\
& =-M_{1} M_{2} \psi+M_{2} M_{1} \psi \\
& =\left(-d \pi\left(y_{1}\right) d \pi\left(y_{2}\right)+d \pi\left(y_{2}\right) d \pi\left(y_{1}\right)\right) \psi \\
& =-\left[d \pi\left(y_{1}\right), d \pi\left(y_{2}\right)\right] \psi \in \mathscr{D},
\end{aligned}
$$

again using (LR4).

This concludes the proof of properties (3.2) and (3.3).

Let $z=x+i y \in \mathfrak{g}_{1}^{*}, x \in \mathfrak{f}_{1}, y \in \mathfrak{m}$. Then, by Stone's theorem, $d \pi(x)=i H$ for a self-adjoint operator $H$, and $\overline{d \pi(y)}=M$ for a second self-adjoint $M$, where, initially, $d \pi(y)$ is only defined on $\mathscr{D}$. Let $\psi_{1}$, $\psi_{2} \in \mathscr{D}$. Then

$$
\begin{aligned}
\left\langle d \pi^{*}(z) \psi_{1}, \psi_{2}\right\rangle & =\left\langle(d \pi(x)+i d \pi(y)) \psi_{1}, \psi_{2}\right\rangle \\
& =\left\langle i H \psi_{1}, \psi_{2}\right\rangle+\left\langle i M \psi_{1}, \psi_{2}\right\rangle \\
& =-\left(\left\langle\psi_{1}, i H \psi_{2}\right\rangle+\left\langle\psi_{1}, i M \psi_{2}\right\rangle\right) \\
& =-\left\langle\psi_{1}, d \pi^{*}(z) \psi_{2}\right\rangle
\end{aligned}
$$

proving (3.4).

Observation 3. The representation $d \pi^{*}$ of $\mathrm{g}_{1}^{*}$ exponentiates to a strongly continuous unitary representation $\pi^{*}$ of the subgroup $G_{1}^{*} \subset G^{*}$. 
Proof. It follows from [8, Lemma A] (which in turn is based on the Extension Theorem $[11,3]$ for hermitian semigroups) that $\mathscr{D}$ consists of analytic vectors for each of the operators $d \pi(y), y \in \mathfrak{m}$. But the operator Lie algebra, $d \pi^{*}\left(g_{1}^{*}\right)$ on $\mathscr{D}$, is generated as a Lie algebra by the set of operators $\{i d \pi(y): y \in \mathfrak{m}\}$ by virtue of Observation 1. Hence, we may apply the Exponentiation Theorem [22], or [5, Thm. 3.1], for Lie algebras of unbounded operators. Using Observation 2, we conclude that the exponentiated representation $\pi^{*}$ is unitary.

It remains to extend $\pi^{*}$ from the subgroup $G_{1}^{*}$ to all of $G^{*}$.

4. Gårding vectors. By virtue of (LR1), the integral

$$
\int_{K} f(k) \pi(k) \psi d k=\pi(f) \psi
$$

is well defined when $f \in C_{c}^{\infty}(K)$, and $\psi \in \mathscr{H}$. Recall that $d k$ denotes the left-invariant Haar measure on $K$. Since $\left.\pi\right|_{K}$ is a unitary representation, the integral is convergent for all $f$ with compact support. If $f$ is also $C^{\infty}$, then $\pi(f) \psi$ is a $C^{\infty}$-vector for the action of $K$, and, in particular, is in the domain $\mathscr{D}(d \pi(x))$ for all $x \in \mathfrak{f}$; and

$$
d \pi(x) \pi(f) \psi=\pi(\tilde{x} f) \psi
$$

where

$$
(\tilde{x} f)(g)=\left.\frac{d}{d t} f(\exp (-t x) \cdot g)\right|_{t=0} .
$$

Observation 4. Let $\psi \in \mathscr{D}$, and let $f \in C_{c}^{\infty}(K)$ have support in the neighborhood $V_{\psi}$ from (2.7) and (LR5). Then $\pi(f) \psi$ is an analytic vector for the operator $d \pi(y)$, cf. (3.5), for all $y \in \mathfrak{m}$. Moreover,

$$
e^{t M} \pi(f) \psi=\int_{K} f(k) \pi(k) \pi\left[e^{t \operatorname{Ad}_{k}-1(y)}\right] \psi d k
$$

where $M=\overline{d \pi(y)}$, and the integral on the right hand side is norm-convergent as a Bochner integral with values in $\mathscr{H}$.

Proof. The proof is essentially contained in [8, §4]. We first note that, by [8, Lemma 0], the function $k \rightarrow \pi\left[e^{t \operatorname{Ad}_{k^{-1}}(y)}\right] \psi$, is continuous on $V_{\psi}$ for all $t$ in an interval $(-\varepsilon, \varepsilon)$ where $\varepsilon$ depends on $\psi$. It follows that the integral on the right hand side of (4.2) is convergent for all $f \in C_{c}^{\infty}(K)$ with support contained in $V_{\psi}$. Since $\left.\pi\right|_{K}$ is unitary, the norm of the integral in (4.2) may be estimated by

$$
\int_{K}|f(k)|\left\|\pi\left[e^{t \operatorname{Ad}_{k-1}(y)}\right] \psi\right\| d k .
$$


On the other hand, we have

$$
e^{t M} \pi(k) \psi=\pi(k) \pi\left[e^{t \operatorname{Ad}_{k}-1(y)}\right] \psi
$$

by virtue of (LR1 and 5) for values of $t$ and $k$ specified as above. It follows that the unbounded self-adjoint operator, $e^{t M}$ passes under the integral sign in (4.1), and (4.2) follows.

The proof that $t \rightarrow e^{t M} \pi(f) \psi$ is analytic for $t \in(-\varepsilon, \varepsilon)$ follows from [8, Lemma 1] and the Extension Theorem for hermitian semigroups, cf. also [8, Lemma A. $2^{0}$ ].

Following [8], we let $\mathscr{D}_{K}$ denote the span of the vectors $\pi(f) \psi$ for $\psi \in \mathscr{D}$, and $f \in C_{c}^{\infty}(K)$ with support of $f$ contained in $V_{\psi}$.

We have seen that $\mathscr{D}_{K}$ is contained in the domain of $d \pi(z)$ for all $z \in \mathrm{g}$. It follows from (3.1) that $\mathscr{D}_{K}$ is also contained in $\mathscr{D}\left(d \pi^{*}(z)\right)$ for all $z \in \mathrm{g}^{*}$.

Lemma 5. Let $C^{1}\left(\mathrm{~g}^{*}\right)$ denote the completion of $\mathscr{D}_{K}$ in the graph norm-topology defined by the operators in $d \pi^{*}\left(\mathrm{~g}^{*}\right)$. Then $C^{1}\left(\mathrm{~g}^{*}\right)$ is invariant under $\pi(k)$ for all $k \in K$.

Proof. Since the operator Lie algebra $d \pi^{*}\left(\mathfrak{g}_{1}^{*}\right)$ exponentiates by Observation 3 in $\S 3$, it is immediate that $C^{1}\left(g_{1}^{*}\right)$ is invariant under $\pi(k)$ for all $k \in K_{1}$. Recall that $K_{1}$ is the closed subgroup of $G_{1}^{*}$ which is generated by $\mathfrak{f}_{1}$.

Let $z \in \mathfrak{g}_{1}^{*}$. Then there are analytic functions $a_{i}(k)$ on $K$ such that

$$
\operatorname{Ad}_{k^{-1}}(z)=\sum_{i=1}^{n} \alpha_{i}(k) y_{i},
$$

where $\left\{y_{i}\right\}_{i=1}^{n}$ is a basis for $\mathrm{g}_{1}^{*}$. It follows that

$$
d \pi^{*}(z) \pi(k) \psi=\sum_{i=1}^{n} \alpha_{i}(k) \pi(k) d \pi^{*}\left(y_{i}\right) \psi
$$

for all $\psi \in \mathscr{D}_{K}$. Although $\mathscr{D}_{K}$ is not invariant under $\pi(k)$, we conclude from (4.4) that the completion $C^{\infty}\left(g_{1}^{*}\right)$ is $\pi(k)$-invariant. The proof of (4.4) in turn follows from (LR1) as in $[8, \S 5]$.

It follows that $\left.\pi\right|_{K}$ restricts to a continuous representation of $K$ on the Fréchet space $C^{\infty}\left(\mathfrak{g}_{1}^{*}\right)$. Since $d \pi(x)$ is defined on $\mathscr{D}_{K}$ for all $x \in \mathfrak{f}$, it follows that $d \pi(x)$ may be viewed as a continuous linear endomorphism in $C^{\infty}\left(g_{1}^{*}\right)$ where continuity is understood in the sense of the Fréchet topology, [9].

5. Extension from $G_{1}^{*}$ to $G^{*}$. By assumption, $\left.\pi\right|_{U \cap K}$ is the restriction of a strongly continuous unitary representation, also denoted $\pi$, of $K$. We now consider $d \pi^{*}(z) \pi(k) \psi$ for $z \in \mathfrak{f} \backslash \mathfrak{f}_{1}, k \in K$, and $\psi \in \mathscr{D}_{K}$. Using 
the above fact, we get $d \pi^{*}(z)=d \pi(z)$, and

$$
d \pi(z) \pi(k) \psi=\pi(k) d \pi\left(\operatorname{Ad}_{k^{-1}}(z)\right) \psi .
$$

When this is combined with formula (4.4), the proof of Lemma 5 may now be completed.

Consider $y \in \mathfrak{m}$, and the self-adjoint operator $M=\overline{d \pi(y)}$.

Lemma 6. For $\psi \in \mathscr{D}_{K}, t \in \mathbf{R}$, and $z \in \mathrm{g}^{*}$, we have

$$
d \pi^{*}(z) e^{i t M} \psi=e^{i t M} d \pi^{*}\left(e^{-i t \mathrm{ad} y}(z)\right) \psi .
$$

Proof. We have seen that $\psi$ in $\mathscr{D}_{K}$ is analytic for $M$. On $\mathscr{D}_{K}$, we consider the norm

$$
\psi \rightarrow\|\psi\|+\sum_{i}\left\|d \pi^{*}\left(z_{i}\right) \psi\right\|=\|\psi\|_{1},
$$

where $\left\{z_{i}\right\}$ is a fixed basis for $\mathrm{g}^{*}$ obtained by completing the basis $\left\{y_{i}\right\}$ for $\mathfrak{g}_{1}^{*}$ considered in $\S 4$.

We proved in [5, Observation 1] that $\psi$ is also analytic for $M$ when $M$ is regarded as an operator with dense domain in the $\|\cdot\|_{1}$-completion of $\mathscr{D}_{K}$. This completion is denoted $C^{1}\left(\mathrm{~g}^{*}\right)$.

In Observation 2 of [5], we proved the estimate

$$
\|(\ell-M) \psi\|_{1} \geq(|\operatorname{Im} \ell|-c)\|\psi\|_{1}
$$

for some positive constant $c$. The estimate holds for $\ell \in \mathbf{C}$, with $|\operatorname{Im} \ell|$ sufficiently large.

It follows from a variant of the Hille-Yosida theorem [1, vol. I, thm. 3.2.22] applied to $C^{1}\left(\mathrm{~g}^{*}\right)$, that $M$, regarded as an operator in $C^{1}\left(\mathrm{~g}^{*}\right)$, generates a strongly continuous one-parameter group on $C^{1}\left(\mathrm{~g}^{*}\right)$, and that this group agrees with $e^{i t M}$ on $\mathscr{H}$.

Combining Lemmas 5 and 6, it follows that, for $|\operatorname{Im} \ell|$ sufficiently large, $\ell \in \mathbf{C}$, the space

$$
\left(\ell-d \pi^{*}(z)\right) \mathscr{D}_{K}
$$

is dense in $C^{1}\left(\mathfrak{g}^{*}\right)$ for all $z \in \mathfrak{g}^{*}$. This is just an application of the "converse" Hille-Yosida theorem to the Banach space $C^{1}\left(\mathrm{~g}^{*}\right)$.

The integrability of the operator Lie algebra $d \pi^{*}\left(\mathrm{~g}^{*}\right)$ now follows from [9, Theorem 9.2] (which is the main theorem on exponentiation of operator Lie algebras from [9]. This result was announced in [10].)

This concludes the proof of the Theorem in $\$ 2$ above. 
REMARK. In special cases, an alternative approach to the extension of $\pi^{*}$ from the subgroup $G_{1}^{*}$ to all of $G^{*}$ may be based instead on [5, Theorem 2.2]. We may apply $\pi^{*}$, viewed as local representation of $G^{*}$, to formula (4.3). Using the argument above, and formula (4.4), we get the estimate

$$
\|d \pi(x) \psi\| \leq \text { Const }\left[\|\psi\|+\sum_{i=1}^{n}\left\|d \pi^{*}\left(y_{i}\right) \psi\right\|\right],
$$

valid for $x \in \mathfrak{f}$, and $\psi \in C^{1}\left(\mathrm{~g}_{1}^{*}\right)=$ the completion of $\mathscr{D}_{K}$ in the graph norm on the right hand side of (4.5).

Our result [5, Theorem 2.2] now implies that $C^{\infty}\left(g_{1}^{*}\right)$ is invariant under $d \pi(x)$, and that the operator family $\left\{d \pi^{*}\left(y_{i}\right): 1 \leq i \leq n\right\}$ analytically dominates $d \pi(x)$. Since $\mathscr{D}$ consists of analytic vectors for this family, it follows that every vector in $\mathscr{D}$ is also analytic for $d \pi(x)$, and integrability of $d \pi^{*}\left(\mathrm{~g}^{*}\right)$ follows from a sècond application of [5, Thm. 3.1], or [22],

Alternatively, it follows that the assumptions in Nelson's extension theorem [14, Theorem 6] are fulfilled, see also [17, Theorem 3.1], and we conclude that $\pi^{*}$ on $G_{1}^{*}$ extends to a unitary representation, also denoted by $\pi^{*}$, of the bigger group $G^{*}$. Moreover, the extended representation is seen to be the exponential of the operator Lie algebra $d \pi^{*}\left(\mathrm{~g}^{*}\right)$, see [14, Lemma 6.3], by analytic domination.

\section{REFERENCES}

[1] O. Bratteli, and D. W. Robinson, Operator Algebras and Quantum Statistical Mechanics, Springer-Verlag, Berlin-N. Y. 1979.

[2] L. Gårding, Vecteurs analytiques dans les représentations des groupes de Lie, Proc. Natl. Acad. Sci. USA, 33 (1947), 331-332.

[3] J. Fröhlich, Unbounded symmetric semigroups on a seperable Hilbert space are essentially selfadjoint, Adv. in Appl. Math., 1 (1980), 237-256.

[4] J. Fröhlich, K. Osterwalder, and E. Seiler, On virtual representations of symmetric spaces and their analytic continuation, Ann. Math., 118 (1983), 461-489.

[5] F. M. Goodman, and P. E. T. Jorgensen, Lie algebras of unbounded derivations, J. Funct. Anal., 52 (1983), 369-384.

[6] R. Goodman, Complex Fourier analysis on a nilpotent Lie group, Trans. Amer. Math. Soc., 160 (1971), 373-391.

[7] S. Helgason, Differential Geometry and Symmetric Spaces, Academic Press, New York 1978.

[8] P. E. T. Jorgensen, Analytic continuation of local representations of symmetric space, preprint 1984, submitted.

[9] P. E. T. Jorgensen, and R. T. Moore, Operator Commutation Relations, D. Reidel Publishing Co., Dordrecht-Boston 1984.

[10] P. E. T. Jorgensen, Perturbation and analytic continuation of group representations, Bull. Amer. Math. Soc., 82 (1976), 921-924. 
[11] A. Klein, and L. J. Landau, Construction of a unique selfadjoint generator for a symmetric local semigroup, J. Funct. Anal., 44 (1981), 121-137.

[12] A. Klein, and L. J. Landau, From the Euclidean to the Poincare group via Osterwalder-Schrader positivity, Comm. Math. Phys., 87 (1983), 469-484.

[13] M. Lüscher, and G. Mack, Global conformal invariance in quantum field theory, Comm. Math. Phys., 41 (1975), 203-234.

[14] E. Nelson, Analytic vectors, Ann. Math., 70 (1959), 572-615.

[15] E. Nelson, Topics in Dynamics, I: Flows, Princeton Univ. Press University Press, Princeton, N. J. 1969.

[16] K. Osterwalder, and R. Schrader, Axioms for Euclidean Green's functions, I, II, Comm. Math. Phys., 31 (1973), 83-112; 42 (1975), 281-305.

[17] R. C. Penney, Non-elliptic Laplace equations on nilpotent Lie groups, Ann. Math., 119 (1984), 309-385.

[18] Harmonically induced representations on nilpotent Lie groups and automorphic forms on nilmanifolds, Trans. Amer. Math. Soc., 260 (1980), 123-145.

[19] R. Schrader, Personal communication, 1985.

[20] Reflection positivity for the complementary, series of $\mathrm{SL}(2 n, \mathbf{C})$, preprint 1985. To appear in Pub. RIMS, Kyoto University.

[21] E. Seiler, Gauge Theories as a Problem of Constructive Quantum Field Theory and Statistical Mechanics, LNP, 159, Springer-Verlag, New York 1982.

[22] J. Simon, On the integrability of representations of finite dimensional real Lie algebras, Comm. Math. Phys., 28 (1972), 39-46.

Received July 3, 1985. Work supported in part by NSF.

THE UNIVERSITY OF IOWA

IOWA CITY, IA 52242 


\title{
PACIFIC JOURNAL OF MATHEMATICS \\ EDITORS
}

\author{
V. S. VARADARAJAN \\ (Managing Editor) \\ University of California \\ Los Angeles, CA 90024 \\ HERBERT CLEMENS \\ University of Utah \\ Salt Lake City, UT 84112 \\ R. FINN \\ Stanford University \\ Stanford, CA 94305
}

\author{
HERMANN FLASCHKA \\ University of Arizona \\ Tucson, AZ 85721
}

RAMESh A. GANGOLLI

University of Washington Seattle, WA 98195

VAUGHAN F. R. JONES

University of California

Berkeley, CA 94720

ROBION KIRBY

University of California

Berkeley, CA 94720
C. C. MOORE

University of California

Berkeley, CA 94720

H. SAMELSON

Stanford University

Stanford, CA 94305

HAROLD STARK

University of California, San Diego

La Jolla, CA 92093

\section{ASSOCIATE EDITORS}

\author{
R. ARENS \\ E. F. BECKENBACH \\ B. H. NeUmanN \\ F. WOLF \\ K. YOSHIDA \\ (1906-1982)

\section{SUPPORTING INSTITUTIONS} \\ UNIVERSITY OF ARIZONA \\ UNIVERSITY OF BRITISH COLUMBIA \\ CALIFORNIA INSTITUTE OF TECHNOLOGY \\ UNIVERSITY OF CALIFORNIA \\ MONTANA STATE UNIVERSITY \\ UNIVERSITY OF NEVADA, RENO \\ NEW MEXICO STATE UNIVERSITY \\ OREGON STATE UNIVERSITY \\ UNIVERSITY OF OREGON \\ UNIVERSITY OF SOUTHERN CALIFORNIA \\ STANFORD UNIVERSITY \\ UNIVERSITY OF HAWAII \\ UNIVERSITY OF TOKYO \\ UNIVERSITY OF UTAH \\ WASHINGTON STATE UNIVERSITY \\ UNIVERSITY OF WASHINGTON
}

The Supporting Institutions listed above contribute to the cost of publication of this Journal, but they are not owners or publishers and have no responsibility for its content or policies.

Mathematical papers intended for publication in the Pacific Journal of Mathematics should be in typed form or offset-reproduced (not dittoed), double spaced with large margins. Please do not use built up fractions in the text of the manuscript. However, you may use them in the displayed equations. Underline Greek letters in red, German in green, and script in blue. The first paragraph must be capable of being used separately as a synopsis of the entire paper. In particular it should contain no bibliographic references. Please propose a heading for the odd numbered pages of less than 35 characters. Manuscripts, in triplicate, may be sent to any one of the editors. Please classify according to the scheme of Math. Reviews, Index to Vol. 39. Supply name and address of author to whom proofs should be sent. All other communications should be addressed to the managing editor, or Elaine Barth, University of California, Los Angeles, California 90024.

There are page-charges associated with articles appearing in the Pacific Journal of Mathematics. These charges are expected to be paid by the author's University, Government Agency or Company. If the author or authors do not have access to such Institutional support these charges are waived. Single authors will receive 50 free reprints; joint authors will receive a total of 100 free reprints. Additional copies may be obtained at cost in multiples of 50 .

The Pacific Journal of Mathematics is issued monthly as of January 1966. Regular subscription rate: $\$ 190.00$ a year (5 Vols., 10 issues). Special rate: $\$ 95.00$ a year to individual members of supporting institutions.

Subscriptions, orders for numbers issued in the last three calendar years, and changes of address should be sent to Pacific Journal of Mathematics, P.O. Box 969, Carmel Valley, CA 93924, U.S.A. Old back numbers obtainable from Kraus Periodicals Co., Route 100, Millwood, NY 10546.

The Pacific Journal of Mathematics at P.O. Box 969, Carmel Valley, CA 93924 (ISSN 0030-8730) publish'es 5 volumes per year. Application to mail at Second-class postage rates is pending at Carmel Valley, California, and additional mailing offices. Postmaster: send address changes to Pacific Journal of Mathematics, P.O. Box 969, Carmel Valley, CA 93924.

PUBLISHED BY PACIFIC JOURNAL OF MATHEMATICS, A NON-PROFIT CORPORATION

Copyright (C) 1986 by Pacific Journal of Mathematics 


\section{Pacific Journal of Mathematics}

\section{Vol. 125, No. $2 \quad$ October, 1986}

Dale Edward Alspach, On $\mathscr{L}_{p, \lambda}$ spaces for small $\lambda \ldots \ldots \ldots \ldots \ldots \ldots 257$

Jong Sook Bae and Sangsuk Yie, Range of Gateaux differentiable operators

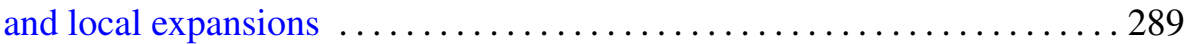

Hubert Berens and Lothar Hetzelt, On accretive operators on $l_{n}^{\infty} \ldots \ldots 301$

Marie-Françoise Bidaut-Véron, Global existence and uniqueness results for singular solutions of the capillarity equation ................. 317

Donald M. Davis and Mark Mahowald, Classification of the stable homotopy types of stunted real projective spaces ................. 335

Aad Dijksma, Heinz K. Langer and Hendrik S. V. de Snoo, Unitary

colligations in $\Pi_{\kappa}$-spaces, characteristic functions and Štraus extensions

Michel Enock and Jean-Marie Schwartz, Algèbres de Kac

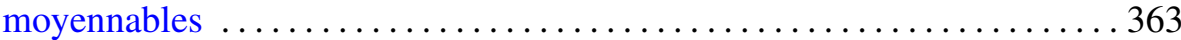

Seppo Granlund, Peter Lindqvist and Olli Martio, Note on the

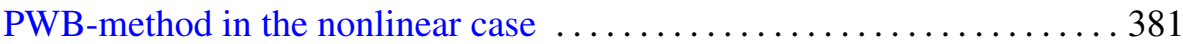

Palle E. T. Jorgensen, Analytic continuation of local representations of Lie

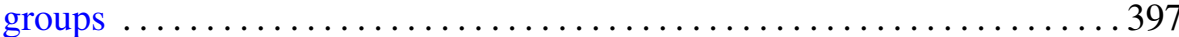

Robert P. Kaufman, Plane curves and removable sets ................409

José M. Montesinos and Wilbur Carrington Whitten, Constructions of two-fold branched covering spaces $\ldots \ldots \ldots \ldots \ldots$.

Benedict Seifert, Highly transitive group actions on trees and normalizing Tits systems

Charles Stuart Stanton, Counting functions and majorization for Jensen

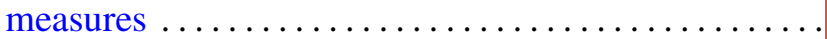

Luen-Fai Tam, On existence criteria for capillary free surfaces without gravity

Zhuocheng Yang, Exposed points of left invariant means 